\title{
Semiclassical analysis of a two-electron quantum dot in a magnetic field: dimensional phenomena
}

\author{
R.G. Nazmitdinov ${ }^{1,2}$, N. S. Simonović ${ }^{1}$, and Jan M. Rost ${ }^{1}$ \\ ${ }^{1}$ Max-Planck-Institut für Physik komplexer Systeme, D-01187 Dresden, Germany \\ ${ }^{2}$ Bogoliubov Laboratory of Theoretical Physics, Joint Institute for Nuclear Research, 141980 \\ Dubna, Russia
}

\begin{abstract}
While the dynamics for three-dimensional axially symmetric two-electron quantum dots with parabolic confinement potentials is in general nonseparable we have found an exact separability with three quantum numbers for specific values of the magnetic field. Furthermore, it is shown that the magnetic properties such as the magnetic moment and the susceptibility are sensitive to the presence and strength of a vertical confinement. Using a semiclassical approach the calculation of the eigenvalues reduces to simple quadratures providing a transparent and almost analytical quantization of the quantum dot energy levels which differ from the exact energies only by a few percent.
\end{abstract}

PACS numbers: 73.21.La, 03.65.Sq, 75.75.+a, 05.45.Mt

Current nanofabrication technology allows one to control the size and shape of quantum dots [1] [3]. Due to the confinement of the electrons in all three spatial directions the energy spectrum is quantized creating excellent experimental and theoretical opportunities to study controlled single-particle and collective dynamics at the atomic scale. For example, depending on the experimental setup, the spectrum of a quantum dot displays shell structure 
[4.5] or follows predictions of random matrix theory (for a review see [6]). Furthermore, it becomes possible to trace the transition from a quantum mechanical to an almost classical regime.

Few-electron quantum dots have attracted special attention [4]:7], since they may provide a natural realization of a quantum bit [1]. The simplest quantum dot with the essential features of more complex systems contains two electrons. Experimental data, including transport measurements [8] and spin oscillations in the ground state under a perpendicular magnetic field [9], have been explained quantum mechanically as a result of the interplay between the two-dimensional lateral confinement potential, electron correlations and the magnetic field [10,11]. While in these experiments effects of the third spatial dimension are somewhat hidden, they naturally come into play with a tilted magnetic field [12]. However, even with a perpendicular magnetic field the vertical confinement has at least two important consequences which will be worked out in the following: first, it changes the magnetic moment and susceptibility with respect to the $2 \mathrm{D}$ results, second, the generically non-separable 3D dynamics becomes separable for certain values of the magnetic field. We will use a semiclassical description which offers a simple and accurate approach to explore the effects of dimensionality in quantum dots. In contrast to a circular (2D) two-electron quantum dot whose classical dynamics is always separable and therefore regular, the corresponding 3D-system with axial symmetry is in general a non-integrable problem with typical features of mixed dynamics (regular/chaotic).

The Hamiltonian for the 3D two-electron quantum dot reads

$$
H=\sum_{j=1}^{2}\left\{\frac{1}{2 m^{*}}\left(\mathbf{p}_{j}-\frac{e}{c} \mathbf{A}_{j}\right)^{2}+\frac{m^{*}}{2}\left[\omega_{0}^{2}\left(x_{j}^{2}+y_{j}^{2}\right)+\omega_{z}^{2} z_{j}^{2}\right]\right\}+V_{C}+H_{\mathrm{spin}},
$$

where $V_{C}=\alpha /\left|\mathbf{r}_{1}-\mathbf{r}_{2}\right|$ is the Coulomb energy $\left(\alpha=e^{2} /\left(4 \pi \varepsilon \varepsilon_{0}\right)\right)$ and $H_{\text {spin }}=g^{*}\left(\mathbf{s}_{1}+\mathbf{s}_{2}\right) \cdot \mathbf{B}$ describes the Zeeman energy. Here $m^{*}$ and $g^{*}$ are the effective electron mass and $g$-factor, respectively, and $\varepsilon$ is the dielectric constant. The confining potential is approximated with a $3 \mathrm{D}$ axially-symmetric harmonic oscillator and $\hbar \omega_{z} \neq \hbar \omega_{0}$ are the energy scales of confinement in the $z$-direction and in the $x y$-plane, respectively. For the typical voltage $\sim 1 \mathrm{~V}$ applied 
to the gate, the confining potential is some $\mathrm{eV}$ deep which is large compared to the few $\mathrm{meV}$ of the confining frequency [2.3]. Hence, the electron wave function is localized close to the minimum of the well which always can be approximated by a parabolic potential. In real samples the electron-electron interaction is usually screened. However, the pure Coulomb interaction should suffice to understand the main features of the system. For the perpendicular magnetic field $(\mathbf{B} \| z)$ we choose a gauge described by the vector $\mathbf{A}=$ $[\mathbf{B} \times \mathbf{r}] / 2=\frac{1}{2} B(-y, x, 0)$. Introducing the relative and center-of-mass coordinates $\mathbf{r}=\mathbf{r}_{1}-\mathbf{r}_{2}$, $\mathbf{R}=\frac{1}{2}\left(\mathbf{r}_{1}+\mathbf{r}_{2}\right)$, the Hamiltonian, Eq.(1) , can be separated into the center-of-mass (CM) $H_{\mathrm{CM}}$ and relative-motion (RM) $H_{\text {rel }}$ terms: $H=H_{\mathrm{CM}}+H_{\text {rel }}+H_{\text {spin }}$. The solution to the CM-Hamiltonian is well known [13] and the effect of the Zeeman energy has been discussed in 10,11]. In the following we will concentrate on the dynamics of $H_{\text {rel }}$.

For our analysis it is convenient to use cylindrical scaled coordinates, $\tilde{\rho}=\rho / l_{0}, \tilde{p}_{\rho}=$ $p_{\rho} l_{0} / \hbar, \tilde{z}=z / l_{0}, \tilde{p}_{z}=p_{z} l_{0} / \hbar$, where $l_{0}=\left(\hbar / \mu \omega_{0}\right)^{1 / 2}$ is the characteristic length of the confinement potential with the reduced mass $\mu=m^{*} / 2$. The strength parameter $\alpha$ of the Coulomb repulsion goes over to $\lambda=2 \alpha /\left(\hbar \omega_{0} l_{0}\right)$. Using the effective mass $m^{*}=0.067 m_{e}$, the dielectric constant $\varepsilon=12$, which are typical for GaAs, and the confining frequency $\hbar \omega_{0}=3$ meV, we obtain $\lambda \approx 3$. Hereafter, for the sake of simplicity, we drop the tilde, i.e. for the scaled variables we use the same symbols as before scaling.

In these variables the Hamiltonian for the relative motion takes a particular simple form (in units of $\left.\hbar \omega_{0}\right)$

$$
\epsilon \equiv \frac{H_{\mathrm{rel}}}{\hbar \omega_{0}}=\frac{1}{2}\left[p_{\rho}^{2}+\frac{m^{2}}{\rho^{2}}+p_{z}^{2}+\left(\frac{\omega_{\rho}}{\omega_{0}}\right)^{2} \rho^{2}+\left(\frac{\omega_{z}}{\omega_{0}}\right)^{2} z^{2}+\frac{\lambda}{\sqrt{\rho^{2}+z^{2}}}\right]-\frac{\omega_{L}}{\omega_{0}} m,
$$

where $m=l_{z} / \hbar, \omega_{L}=e B / 2 m^{*} c$ is the Larmor frequency and

$$
\omega_{\rho}=\left(\omega_{L}^{2}+\omega_{0}^{2}\right)^{1 / 2}
$$

is the effective confinement frequency in the $\rho$-coordinate which depends through $\omega_{L}$ on the magnetic field.

Due to the cylindrical symmetry, the $z$-component $l_{z} \equiv p_{\phi}$ of the angular momentum is conserved and the motion in $\phi$ is separated from the motion in the $(\rho, z)$-plane. Since the 
Coulomb term couples the two coordinates, the problem is in general non-integrable which is reflected in the Poincaré sections shown in Fig.1 for increasing magnetic field. The chosen ratio $\omega_{z} / \omega_{0}=3$ is of the same order of magnitude as in the experiment [12]. For $\omega_{L}=0$ and small values of $m$ the motion is mainly chaotic (see Fig.1a). With the magnetic field the frequency of oscillations along the $\rho$-coordinate can be controlled which leads to qualitatively different dynamical situations (Fig.1b-d). For equal effective confinement frequencies $\omega_{\rho}^{2}=$ $\omega_{z}^{2}$, the Hamiltonian Eq.(2) becomes separable in spherical coordinates and the dynamics is integrable (Fig.1c). For two other limiting cases, the dynamics is nearly integrable, namely in the limit $m \rightarrow \infty$ and for $\omega_{z} \rightarrow \infty$. The latter case represents a two-dimensional quantum dot, classically, we have $p_{z}, z \rightarrow 0$ in this limit.

The semiclassical quantization of the circular $2 D$ quantum dot is particularly simple since it reduces to a $1 \mathrm{D} \mathrm{WKB}$ quantization of the $\rho$-motion due to the separability of the problem. For given $m$ and $p_{z}=z=0$ the momentum $p_{\rho}$ determined from Eq.(2) enters the action integral

$$
I_{\rho}=\frac{\hbar}{2 \pi} \oint p_{\rho} d \rho=\frac{\hbar}{\pi} \int_{\rho_{\min }}^{\rho_{\max }}\left|p_{\rho}\right| d \rho
$$

with the turning points $\rho_{\min }, \rho_{\max }$ as the positive roots of equation $p_{\rho}(\rho)=0$. The WKB quantization conditions

$$
I_{\rho}(\epsilon)=\hbar\left(n_{\rho}+\frac{1}{2}\right), \quad n_{\rho}=0,1, \ldots, \quad m=0, \pm 1, \ldots
$$

determine the energy levels. For non-interacting electrons $(\lambda=0)$ the analytical calculation of the action integral leads to the (quantum mechanically exact) eigen-energies

$$
\epsilon=\sqrt{1+\left(\frac{\omega_{L}}{\omega_{0}}\right)^{2}}\left(2 n_{\rho}+|m|+1\right)-\frac{\omega_{L}}{\omega_{0}} m
$$

which are the well known Fock-Darwin energies [13]. For $\lambda \neq 0$, we calculate the action integral Eq.(44) numerically with a few iterations to determine the quantum eigenvalues.

The energy spectra for non-interacting and interacting electrons are shown in Fig.2. In the interacting case the semiclassical result, although not exact (the error is less than 1\%), reproduces very well the quantum mechanical results [11, [10]. 
Turning now to the 3D quantum dot we have seen that the dynamics is separable for $\omega_{z}^{2}=\omega_{\rho}^{2} \equiv \omega_{L}^{* 2}+\omega_{0}^{2}$ and the Hamiltonian Eq. (2) in scaled spherical coordinates takes the form

$$
\epsilon=\frac{1}{2}\left\{p_{r}^{2}+\left(\frac{\omega_{z}}{\omega_{0}}\right)^{2} r^{2}+\frac{\lambda}{r}+\frac{(\mathbf{l} / \hbar)^{2}}{r^{2}}\right\}-\frac{\omega_{L}^{*}}{\omega_{0}} m .
$$

In this case the square of the total angular momentum $\mathbf{l}^{2}$ is an additional integral of motion. Therefore, the classical dynamics reduces again to a one-dimensional, radial problem. Using Eq.(7) and calculating the action integral for the radial motion analogous to that in Eq.(四) (i.e. with $r$ instead of $\rho$ ), we obtain the energy levels from the standard WKB quantization conditions

$$
\begin{aligned}
& I_{r}(\epsilon)=\hbar\left(n_{r}+\frac{1}{2}\right), \quad|\mathbf{l}|=\hbar\left(l+\frac{1}{2}\right), \\
& n_{r}, l=0,1, \ldots, \quad m=0, \pm 1, \ldots, \pm l
\end{aligned}
$$

Note that it is only the magnetic field which generates the spherical symmetry of the problem and therefore its separability leading to three good quantum numbers $n_{r}, l$ and $m$.

In the general case of an axially symmetric $3 D$ quantum dot we have non-integrable motion and a semiclassical quantization is neither straight forward nor does it give results which allow for a simple understanding of the dynamics. Therefore, we make use of the fact that in real samples the confining potential in the $z$-direction is much stronger than in the $x y$-plane which allows us to analyze the $3 \mathrm{D}$ non-integrable system with the 'removal of resonances' method (RRM) [14]. To lowest order the RRM consists of averaging the Hamilton function over the fastest angle of the unperturbed motion $(\lambda=0)$ after rewriting coordinates and momenta in terms of action-angle variables $\left(J_{\rho}, J_{z}, \theta_{\rho}, \theta_{z}\right)$ :

$$
\begin{aligned}
& \rho^{2}=\frac{\omega_{0}}{\omega_{\rho}}\left(2 j_{\rho}+|m|-2 \sqrt{j_{\rho}\left(j_{\rho}+|m|\right)} \cos 2 \theta_{\rho}\right), \\
& z^{2}=\frac{2 j_{z} \omega_{0}}{\omega_{z}} \sin ^{2} \theta_{z},
\end{aligned}
$$

and $p_{\rho}=\dot{\rho}, p_{z}=\dot{z}$. Here, $j_{z}=J_{z} / \hbar$ and $j_{\rho}=J_{\rho} / \hbar$. If $\omega_{z}>\omega_{\rho}$ one averages over the angle $\theta_{z}=\omega_{z} t$. As a result, the motion effectively decouples into an unperturbed motion in 
the $z$-coordinate governed by the potential $\left(\omega_{z} / \omega_{0}\right)^{2} z^{2} / 2$ and into the relative motion in the $\rho$-coordinate governed by the effective potential

$$
V_{\mathrm{eff}}\left(\rho, j_{z}\right)=\frac{1}{2}\left(\frac{\omega_{\rho}}{\omega_{0}}\right)^{2} \rho^{2}+\frac{m^{2}}{2 \rho^{2}}+\frac{\lambda}{\pi \rho} K\left(-2 \frac{\omega_{0}}{\omega_{z}} \frac{j_{z}}{\rho^{2}}\right),
$$

where $K$ is the first elliptic integral. Hence, the effective Hamiltonian reads

$$
\epsilon=\frac{p_{\rho}^{2}}{2}+V_{\mathrm{eff}}-\frac{\omega_{L}}{\omega_{0}} m+\frac{\omega_{z}}{\omega_{0}} j_{z}
$$

Applying a similar procedure as in the 2D case, we calculate the action integral numerically. The momentum $p_{\rho}$ is determined from Eq.(12) and the turning points $\rho_{\min }, \rho_{\max }$ are as usual the (positive) roots of the equation $p_{\rho}(\rho)=0$. Finally, the WKB-quantization conditions

$$
\begin{aligned}
& I_{\rho}(\epsilon)=\hbar\left(n_{\rho}+\frac{1}{2}\right), \quad j_{z}=n_{z}+\frac{1}{2} \\
& n_{\rho}, n_{z}=0,1,2, \ldots, \quad m=0, \pm 1, \pm 2, \ldots
\end{aligned}
$$

determine the energy levels.

Comparing the exact results for eigen-energies for the spherical case $\omega_{z} / \omega_{\rho}=1$ we found good agreement even for large values of the magnetic field (Fig.3a) although RRM is expected to work best for $\omega_{\rho} / \omega_{z}<1$. Without magnetic field we have $\omega_{\rho} / \omega_{z}=1 / 3$ which means that the motion in $z$ and $\rho$ approximately decouples justifying the widely used 2D approximation. This is also reflected in the small difference between $2 \mathrm{D}$ and $3 \mathrm{D}$ results (compare Fig.2b with Fig.3a at $\omega_{L}=0$ ). Turning on the magnetic field increases the coupling of the dynamics in $\rho$ and $z$ which allows the two electrons eventually to access the full 3D space. As a consequence, the electrons can avoid each other more effectively and the Coulomb interaction has a smaller effect on the $3 \mathrm{D}$ spectrum than on the $2 \mathrm{D}$ spectrum which is most clearly visible for the $m=0$ energies, see Fig.3a. We can understand this effect quantitatively by averaging the elliptic integral in Eq.(11) over the unperturbed $(\lambda=0)$ motion in $\rho$. It gives rise to an effective charge in the Coulomb interaction $V_{C} \approx \lambda_{\text {eff }} / 2 \rho$, where

$$
\lambda_{\mathrm{eff}}=\frac{2 \lambda}{\pi^{2}} \int_{0}^{\pi} K\left(-\frac{\omega_{\rho} / \omega_{z}}{1+|m|-\sqrt{1+2|m|} \cos 2 \theta_{\rho}}\right) d \theta_{\rho}
$$


for $n_{\rho}=n_{z}=0 \quad\left(j_{\rho}=j_{z}=1 / 2\right)$. The 3D energy quantized with this effective charge for the repulsion is close to the full interaction (dotted line in Fig.3a).

The effective charge $\lambda_{\text {eff }} / \lambda$ as a function of $\omega_{\rho} / \omega_{z}$ for different $m$ is shown in Fig.3b. The maximum repulsion at $\omega_{\rho} / \omega_{z}=0$ corresponds with $\omega_{z} \rightarrow \infty$ to the $2 \mathrm{D}$ case. The $3 \mathrm{D}$ case without magnetic field starts for our parameters $\omega_{\rho} / \omega_{z}=1 / 3$ at some value $\lambda_{\text {eff }} / \lambda<1$ which decreases further for increasing $\omega_{\rho} / \omega_{z}$, i.e., increasing magnetic field. This explains quantitatively through the effective charge the difference of the effect of a magnetic field on a quantum spectrum in $2 \mathrm{D}$ and $3 \mathrm{D}$ cases. However, this difference becomes weaker for larger $m$ as it is seen in Fig.3b.

Although the ground state as a function of the magnetic field is formed piecewise by levels of increasing $m$ and alternating singlet-triplet character (see Fig.3a) the magnetic properties of the ground state nevertheless reveal the dimensional difference between 2D and $3 \mathrm{D}$. At temperature $T=0$ the dot is in the ground state and the magnetic moment and the magnetic susceptibility are defined by $\mu_{\mathrm{mag}}=-\partial E_{\mathrm{gr}} / \partial B$ and $\chi=\partial \mu_{\mathrm{mag}} / \partial B$, respectively. Both quantities exhibit discontinuities as a function of the magnetic field due to the symmetry changes (with respect to $m$ and spin). We find that these discontinuities shift when going from the 2D quantum dot to the 3D case as shown in Fig.4.

By relaxing the restriction of two dimensions for a quantum dot and working in the physical three-dimensional space we have investigated physical examples of non-integrable systems close to integrability. For this situation the RRM method is naturally justified, since the confining frequencies in quantum dots obey the condition $\hbar \omega_{z} \gg \hbar \omega_{0}$. The WKBapproach provides a simple and transparent way to calculate the spectrum of the 3D twoelectron quantum dot even for small values of the quantum numbers. We have found that at specific values of the magnetic field $\omega_{L}^{*}=\sqrt{\omega_{z}^{2}-\omega_{0}^{2}}$ an axially-symmetric quantum dot exhibits spherical symmetry and its dynamics becomes completely separable with three integrals of motion and three corresponding quantum numbers. We have shown that the confinement in the $z$-direction, neglected in the 2D description of quantum dots, does have an influence on the spectrum and consequently also on magnetic properties of the dot. In 
fact, by changing the confining frequency in the $z$-direction only slightly one can increase or decrease the magnetic moment and the susceptibility, i.e. one can control the magnetic properties of the two-electron quantum dot. 


\section{REFERENCES}

[1] R. Turton, The Quantum Dot. A Journey into Future Microelectronics (Oxford University Press, New York, 1995)

[2] D. Bimberg, M. Grundmann, and N.N. Ledentsov, Quantum Dot Heterostructures (John Willey \& Sons, New York, 1998)

[3] T. Chakraborty, Quantum Dots (Elsevier, Amsterdam, 1999)

[4] L.P. Kouwenhoven, D.G. Austing and T. Tarucha, Rep.Prog.Phys. 64, 701 (2001)

[5] M. Macucci, K. Hess, and G.J. Iafrate, Phys. Rev. B 48, 17354 (1993); J. Appl. Phys. 77, 3267 (1995); A. Wojs and P. Hawrylak, Phys.Rev. B 53, 10841 (1996); W.D. Heiss and R.G. Nazmitdinov, Phys. Lett. A 222, 309 (1996); Phys.Rev. B55, 16310 (1997); JETP Letters, 68, 915 (1998)

[6] Y. Alhassid, Rev.Mod.Phys. 72, 895 (2000)

[7] P.A. Maksym, H. Imamura, G.P. Mallon and H. Aoki, J.Phys.: Condens. Matter 12, R299 (2000)

[8] Bo Su, V.J. Goldman, J.E. Cunningham, Phys.Rev.B 46, 7644 (1992)

[9] R.C. Ashoori, H.L. Stormer, J.S. Weiner, L.N. Pfeiffer, K.W. Baldwin and K.W. West, Phys. Rev. Lett., 71, 613 (1993); R.C. Ashoori, Nature (London) 379, 413 (1996)

[10] U. Merkt, J. Huser and M. Wagner, Phys.Rev.B 43, 7320 (1991); M. Wagner, U. Merkt and A.V. Chaplik, Phys. Rev.B 45, 1951 (1992)

[11] M. Dineykhan and R.G. Nazmitdinov, Phys. Rev.B 55, 13707 (1997); J. Phys.: Condens.Matter 11, L83 (1999)

[12] B. Meurer, D. Heitmann, and K. Ploog, Phys.Rev. B 48, 11488 (1993)

[13] V. Fock, Z. Phys. 47, 446 (1928); C.G. Darwin, Proc.Cambridge Philos.Soc. 27, 86 
[14] A.J. Lichtenberg and M.A. Lieberman, Regular and Chaotic Dynamics, Second Edition (Springer-Verlag, New York, 1992)

\section{Figure captions:}

Fig.1. Poincaré surfaces of sections $z=0, p_{z}>0$ of the relative motion for the axiallysymmetric $3 \mathrm{D}$ two-electron quantum $\operatorname{dot}\left(\omega_{z} / \omega_{0}=3, \lambda=3, m=0, \epsilon=5\right)$ in the magnetic field for: (a) $\omega_{L}=0$, (b) $\omega_{L} / \omega_{0}=2.5$, (c) $\omega_{L} / \omega_{0}=\sqrt{8}$ and (d) $\omega_{L} / \omega_{0}=3.3$. The section (c) indicates that for the corresponding value of the magnetic field the system is integrable.

Fig.2. The energy spectrum of a circular $2 \mathrm{D}$ quantum dot (in units $\hbar \omega_{0}$ ) as a function of the ratio $\omega_{L} / \omega_{0}$ for $n_{\rho}=0$ and $m=0, \ldots, 9$ in the cases: (a) $\lambda=0$ and (b) $\lambda=3$.

Fig.3. (a) The comparison between energy levels (in units $\hbar \omega_{0}$ ) of the axially-symmetric $3 \mathrm{D}$ quantum dot with $\omega_{z} / \omega_{0}=3$ and $\lambda=3$ for $n_{\rho}=n_{z}=0$ and $m=0, \ldots, 9$ obtained using the RRM (full lines) and exact results for the spherical case (circles). The inset shows a good agreement between the RRM and the exact results. The dashed and dotted lines display the energy level with $m=0$ for the $2 \mathrm{D}$ and $3 \mathrm{D}$ cases with $\lambda_{\text {eff }}$ at $\omega_{0} / \omega_{z}=0$ and $1 / 3$, respectively. (b) The dependence of the effective strength of the Coulomb interaction $\lambda_{\text {eff }} / \lambda$ on the ratio $\omega_{\rho} / \omega_{z}$.

Fig.4. Magnetic moments $\mu_{\text {mag }}$ (a) in the units of effective Bohr magneton $\mu_{B}^{*}=$ $\left(m_{e} / m^{*}\right) \mu_{B}$ and the magnetic susceptibility $\chi(\mathrm{b})$ for the 2D (dashed lines) and 3D (full lines) cases as a function of the magnetic field strength (in $\omega_{L} / \omega_{0}$-units). We use the same parameters as in Figs.2,3. 
This figure "fig1.gif" is available in "gif" format from: http://arxiv.org/ps/cond-mat/0111381v2 

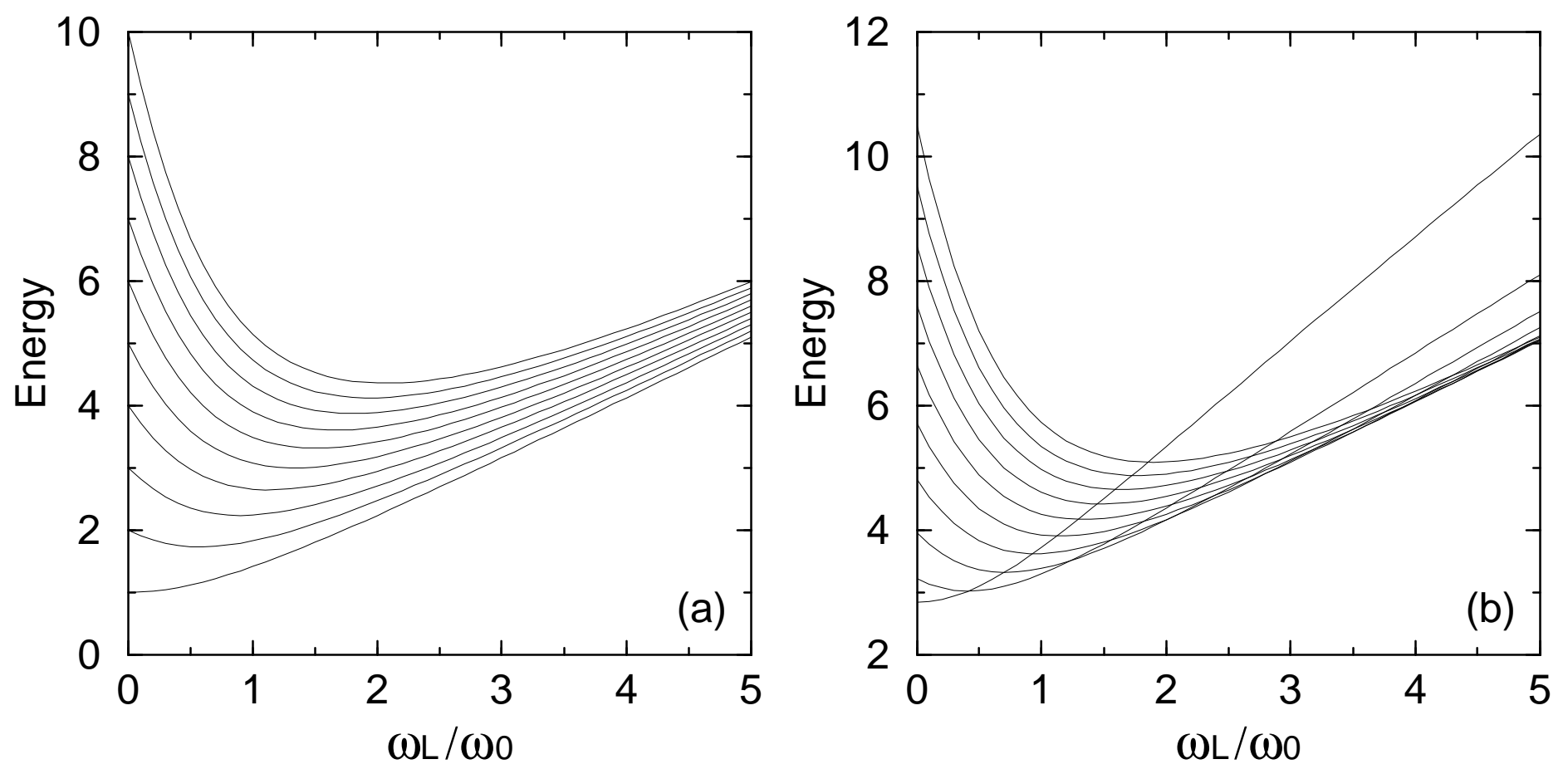

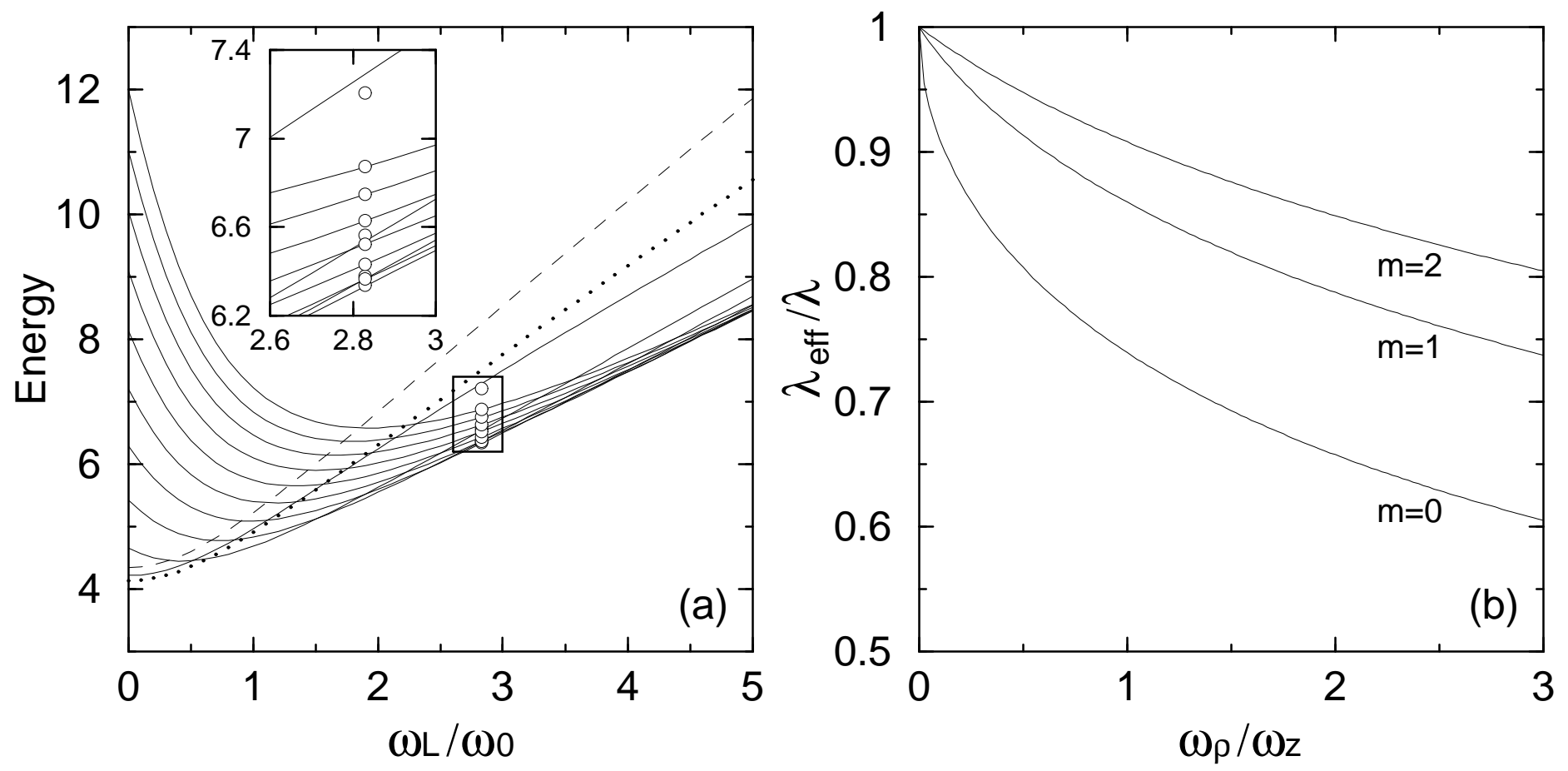


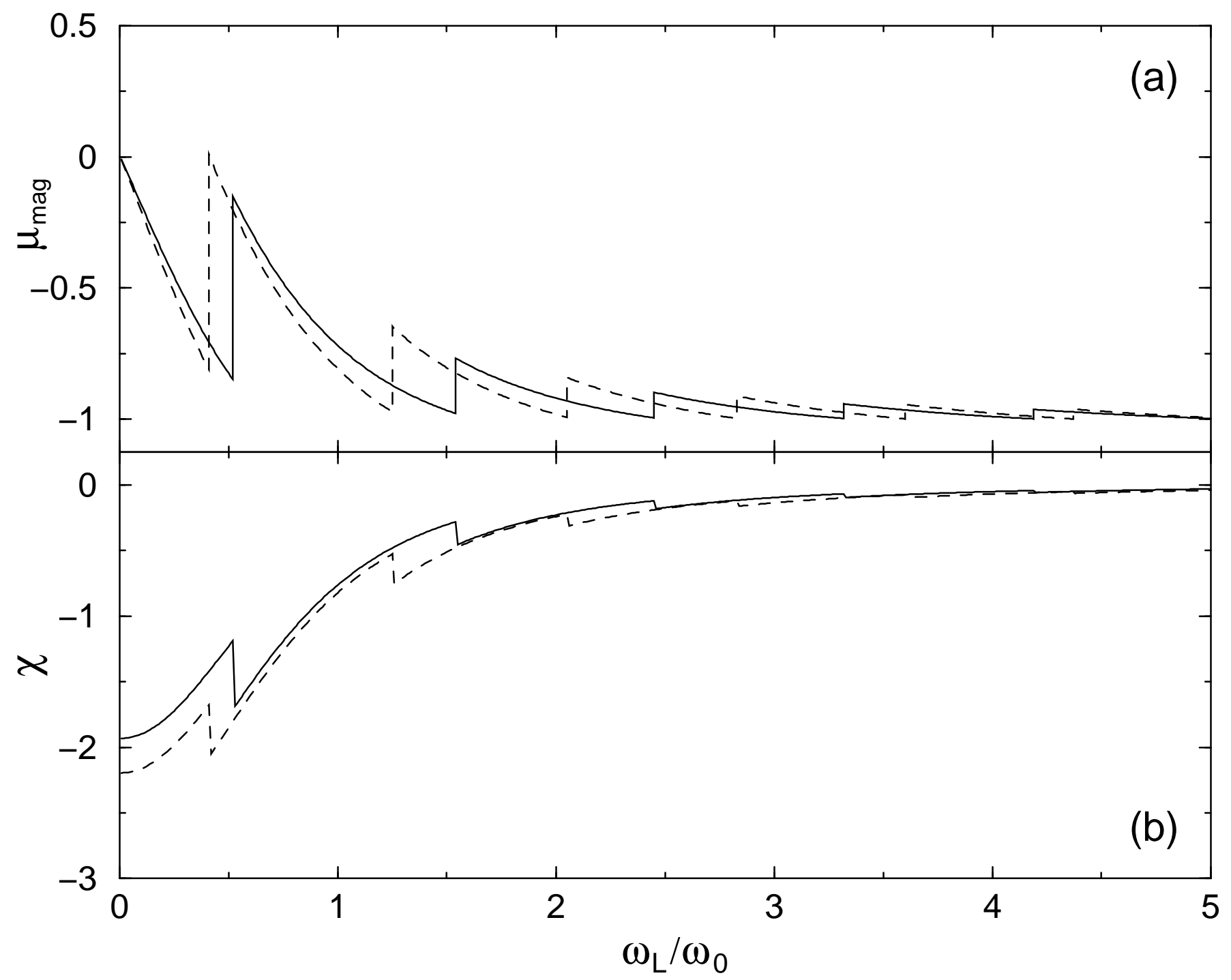

